\title{
Differential response of internal carotid artery blood flow to cardiac output changes
}

\author{
${ }^{1}$ Maria Skytioti, ${ }^{2}$ Signe Søvik, ${ }^{1}$ Maja Elstad \\ ${ }^{1}$ Division of Physiology, Institute of Basic Medical Sciences, University of Oslo \\ ${ }^{2}$ Dept. of Anaesthesia and Intensive Care, Akershus University Hospital, Oslo, Norway
}

\section{Background and Goal of Study}

During central hypovolemia induced by lower body negative pressure, reduced cardiac output (CO) affects cerebral blood flow despite a stable mean arterial blood pressure (MAP) ${ }^{1}$. We studied the response of internal carotid artery blood flow (ICABF) to non-pharmacological $\mathrm{CO}$ elevations in healthy humans.

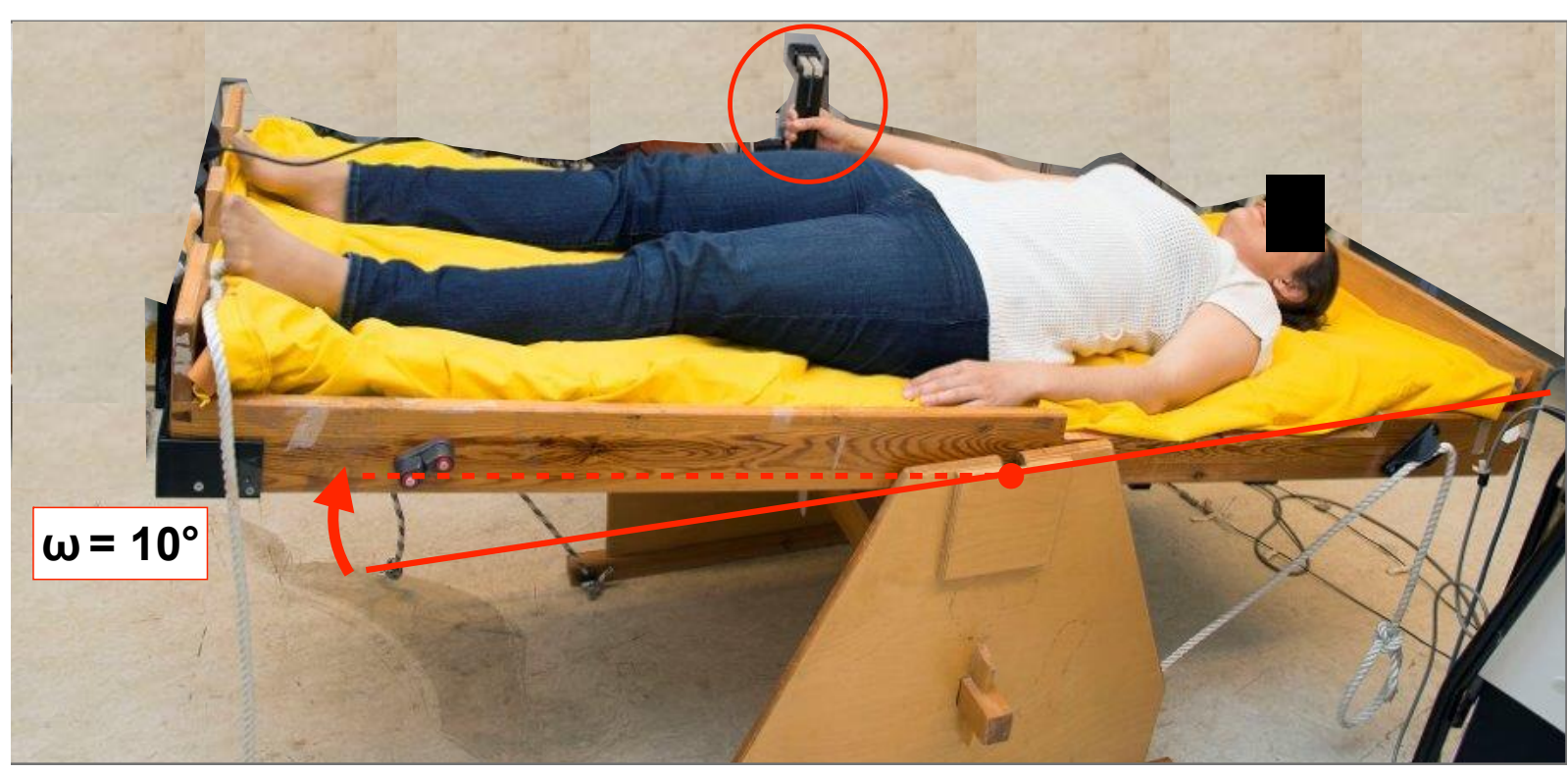

Figure 1. Photo of the laboratory setup. The subject lies on the tilt bed and performs a right hand isometric handgrip during a $10^{\circ}$ head-down tilt.

\section{Materials and Methods}

Internal carotid artery (ICA) blood velocity was measured by Doppler ultrasound in 13 healthy subjects in the horizontal position and during a $10^{\circ}$ head-down tilt (central volume loading), alone or combined with a 3 -min isometric handgrip of $30 \%$ maximal voluntary contraction. Heart rate (HR), MAP, CO (Finometer), and end-tidal $\mathrm{CO}_{2}\left(\mathrm{ETCO}_{2}\right)$ were recorded. ICABF was calculated beat-by-beat. The ICABF response to $\mathrm{CO}$ changes was modelled by linear mixed-effects multiple regression. $\mathrm{ETCO}_{2}, \mathrm{MAP}$ and $\mathrm{CO}$ were continuous covariates. Subject Identity was entered as random effect in the model.

Table 1. Cardiovascular and respiratory variables during different experimental conditions.

\begin{tabular}{|c|c|c|c|c|}
\hline & \multicolumn{2}{|c|}{ Horizontal } & \multicolumn{2}{|c|}{ Central volume loading } \\
\hline & Rest & Handgrip & Rest & Handgrip \\
\hline $\begin{array}{c}\text { HR } \\
(\mathrm{bpm})\end{array}$ & $\begin{array}{c}58 \\
(52,59)\end{array}$ & $\begin{array}{c}64^{*} \\
(57-67)\end{array}$ & $\begin{array}{c}58 \\
(53-60)\end{array}$ & $\begin{array}{c}67^{*} \\
(58-71)\end{array}$ \\
\hline $\begin{array}{c}\text { MAP } \\
(\mathrm{mmHg})\end{array}$ & $\begin{array}{c}73 \\
(67,77)\end{array}$ & $\begin{array}{c}84^{*} \\
(80,87)\end{array}$ & $\begin{array}{c}72 \\
(69,74)\end{array}$ & $\begin{array}{c}85^{*} \\
(78,88)\end{array}$ \\
\hline $\begin{array}{l}\text { ICABF } \\
\text { (ml/min) }\end{array}$ & $\begin{array}{c}260 \\
(188,304)\end{array}$ & $\begin{array}{c}282 \\
(200,323)\end{array}$ & $\begin{array}{c}265 \\
(190,287)\end{array}$ & $\begin{array}{c}257 \\
(182,295)\end{array}$ \\
\hline $\begin{array}{c}\text { CO } \\
\text { (L/min) }\end{array}$ & $\begin{array}{c}5.2 \\
(4.0,5.8)\end{array}$ & $\begin{array}{c}5.9^{*} \\
(4.6,6.6)\end{array}$ & $\begin{array}{c}5.4^{*} \\
(4.2,6.0)\end{array}$ & $\begin{array}{c}6.4^{*} \\
(4.8,7.0)\end{array}$ \\
\hline $\begin{array}{c}\mathrm{ETCO}_{2} \\
(\mathrm{kPa})\end{array}$ & $\begin{array}{c}4.4 \\
(4.0,4.6)\end{array}$ & $\begin{array}{c}4.2 \\
(3.9,4.4)\end{array}$ & $\begin{array}{c}4.4 \\
(4.1,4.6)\end{array}$ & $\begin{array}{c}4.2 \\
(4.0,4.4)\end{array}$ \\
\hline $\begin{array}{l}\text { Medians } \\
\text { Lehmann's } \\
p<0.01, W\end{array}$ & $\begin{array}{l}\text { and } 95 \% \\
\text { estimate. }\end{array}$ & $\begin{array}{l}\text { nfidence } \\
\text { gnificance }\end{array}$ & vals calcu & $\begin{array}{l}\text { by Hodges } \\
\text { rizontal rest }\end{array}$ \\
\hline
\end{tabular}

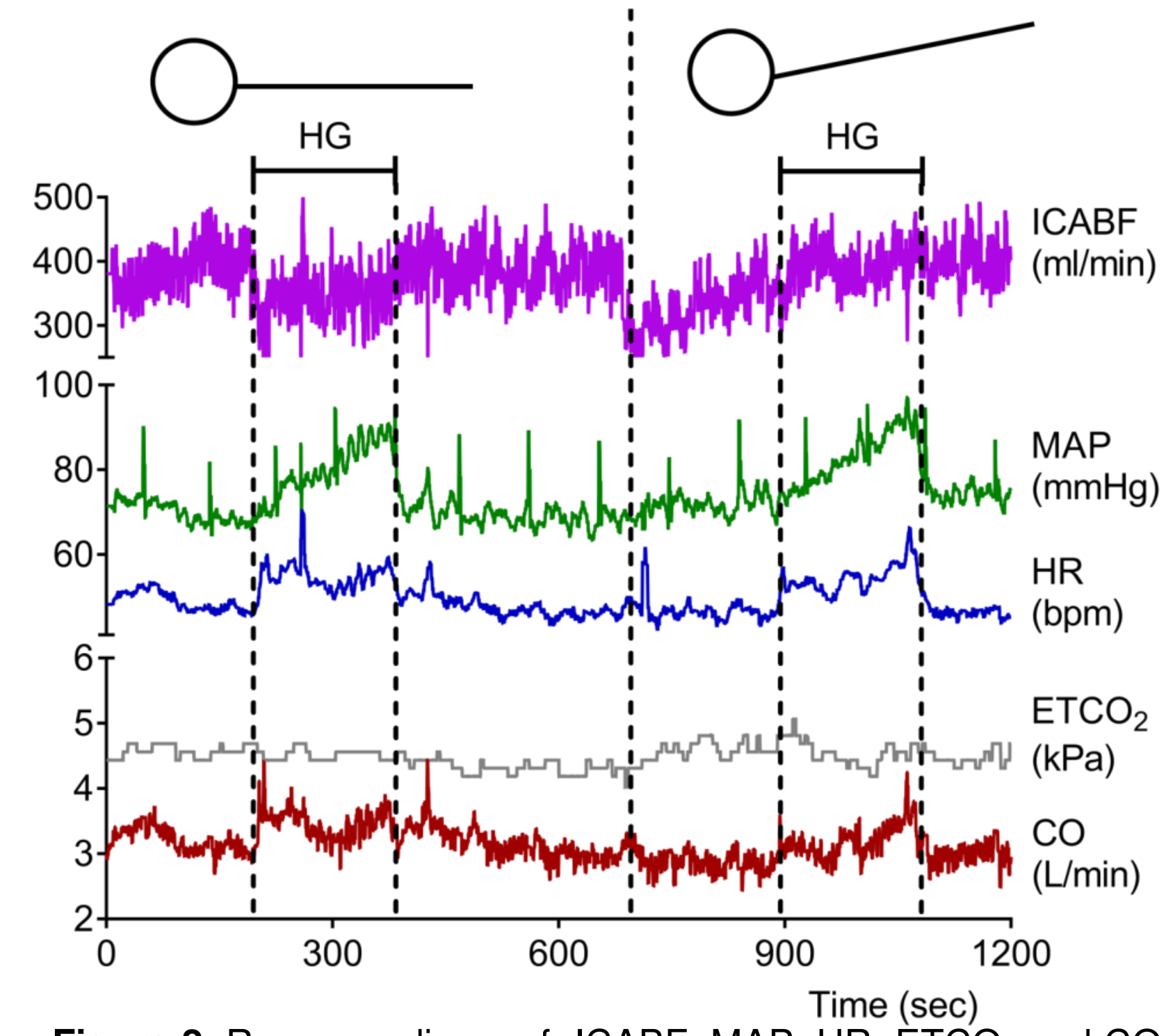

Figure 2. Raw recordings of ICABF, MAP, $\mathrm{HR}, \mathrm{ETCO}_{2}$ and $\mathrm{CO}$ from one subject. HG: handgrip.

\section{Results}

Mixed-model analysis showed that neither CO nor MAP increases induced any ICABF response during central volume loading, with or without isometric handgrip ( $p>0.2$, Figure $3 B, 3 C$, Table 1). In contrast, $\mathrm{CO}$ reduction during central hypovolemia was found to reduce ICABF ( $p=0.01$, Figure $3 A$, previously published finding ${ }^{1}$ ).
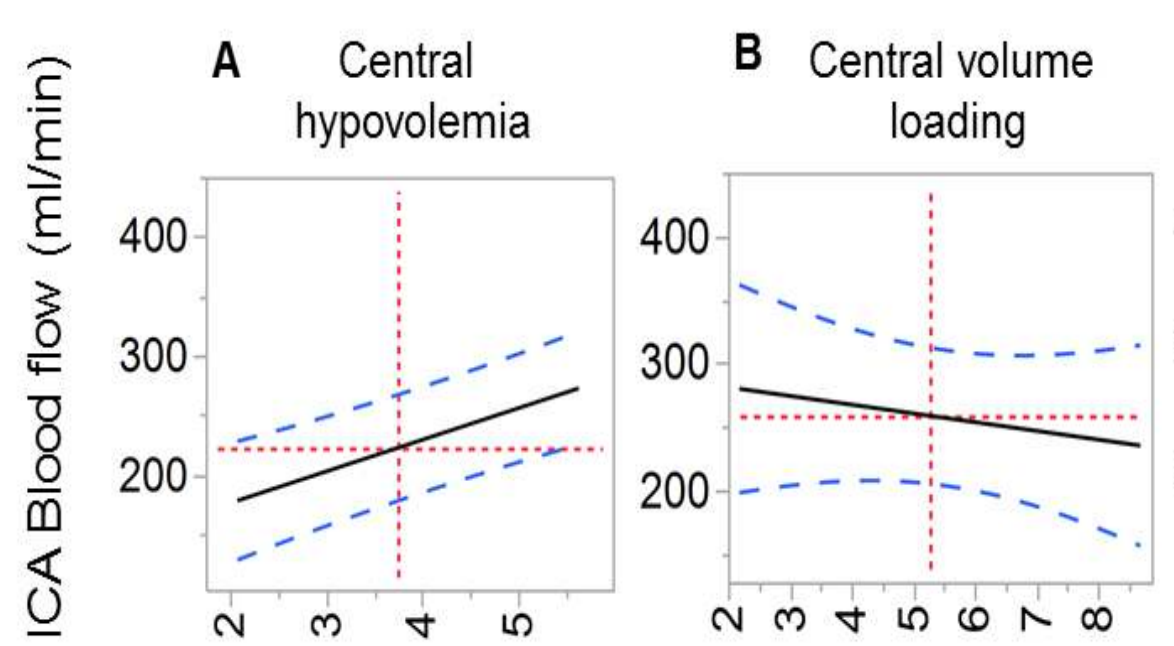

\section{Central volume} loading+Handgrip

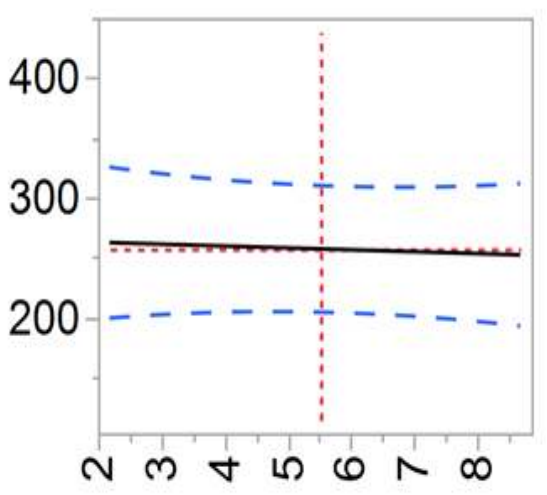

Cardiac Output (L/min)

Figure 3. Absence of relationship between cardiac output and internal carotid artery (ICA) blood flow (B) during central volume loading alone or $(C)$ combined with handgrip. (A) Linear relationship between ICA blood flow and cardiac output during central hypovolemia ${ }^{1}$.

\section{Discussion}

Cerebral vasoconstriction due to sympathetic activation might have counteracted the increase in $\mathrm{CO}$ during central volume loading and/or isometric handgrip, while it may have contributed to ICABF decrease during central hypovolemia. Thus, in contrast to during central volume depletion and $\mathrm{CO}$ reduction ${ }^{1}$, cerebrovasculature may compensate for $\mathrm{CO}$ elevations due to central volume loading and static exercise. 